\begin{tabular}{lll} 
Abstract P31 Table 1 & Distribution of lesions \\
\hline \multicolumn{3}{c}{ KBlenorrhagica } \\
\hline 100\% Weightbearing & $10 / 25(40 \%)$ & $2^{\circ}$ Syphilis \\
$>90 \%$ Weightbearing & $8 / 25(32 \%)$ & 0 \\
$>70 \%$ Weightbearing & $5 / 25(20 \%)$ & 0 \\
Other/Mixed & $2 / 25(8 \%)$ & $5 / 50(10 \%)$ \\
$>70 \%$ Non-Weightbearing & 0 & $7 / 50(14 \%)$ \\
$>90 \%$ Non-Weightbearing & 0 & $12 / 50(24 \%)$ \\
$100 \%$ Non-Weightbearing & 0 & $30 / 50(60 \%)$ \\
\hline
\end{tabular}

Discussion/conclusion The plantar rash of $2^{\circ} \mathrm{Syph}$ is probably seen mostly in thinner areas of arch-of-foot epithelium because vasculitis is hidden under the thickly keratinised weightbearing sole. Any rash covering both areas must raise the possibility of an alternative or double diagnosis or an especially florid presentation.

\section{P32 SURVEY OF KNOWLEDGE ABOUT GONORRHOEA IN PATIENTS WITH GONORRHOEA}

1,2Lauren Amor*, ${ }^{2}$ Joanna Peters, ${ }^{2}$ Angela Dunne, ${ }^{2}$ John Paul, ${ }^{2}$ Gillian Dean, ${ }^{2}$ Fiona Cresswell. 'Brighton and Sussex Medical School, Brighton, East Sussex, UK; ${ }^{2}$ Brighton and Sussex University Hospitals, Brighton, East Sussex, UK

\subsection{6/sextrans-2015-052126.76}

Background/introduction Gonorrhoea is a public health problem due to rising incidence and antimicrobial resistance. Health education is a proven health intervention. Planning interventions requires understanding of views of target groups.

Aim(s)/objectives Describe subjective knowledge of gonorrhoea and preferred methods of health education in individuals presenting with gonorrhoea. Identify differences across specified age groups and sexual orientation.

Methods A prospective study recruited 121 individuals with gonorrhoea. Participants completed a questionnaire. Data from questionnaires were anonymised and analysed.

Results Demographic aspects of this study are presented in a separate abstract. Subjective knowledge about gonorrhoea increases with age and is similar in MSM and heterosexuals. Popularity of mobile Apps decreases with age; 43.8\% of 18-24 year olds, compared with $25 \%$ of over 44 year olds, regard them as beneficial educational tools. 64\%, regardless of age or orientation, favour websites as the educational tool for the public. MSM prefer information on posters in social venues $(50.7 \%$ vs $27.3 \%$ in heterosexuals) or by face-to-face interactions with healthcare workers $(52.2 \%$ vs $23.3 \%$ in heterosexuals). Heterosexuals favoured more information in schools compared to MSM (50\% vs 33\%).

Discussion/conclusion Web-based information was the preferred education method across age groups and sexualities. Posters in bars and clubs would be a good way to target MSM especially as these venues have already been identified as high risk venues associated with GC infection. Future mobile App development should target $18-24$ yr olds.

\section{P33 IS TEST OF CURE NECESSARY AFTER DOXYCYCLINE THERAPY FOR RECTAL CHLAMYDIA TRACHOMATIS INFECTION?}

${ }^{1}$ Emma Hathorn, ${ }^{2}$ Daniel Ward*, ${ }^{1}$ Penny Goold. ' Whittall Street Clinic, University Hospitals Birmingham NHS Foundation Trust, Birmingham, UK; ${ }^{2}$ University of Birmingham, Birmingham, UK

10.1136/sextrans-2015-052126.77
Background/introduction We reported a significantly higher treatment failure rate with azithromycin for the treatment of rectal Chlamydia trachomatis (CT) when compared to doxycycline (26.2\% vs. $0 \%, \mathrm{p}=0.0025)$. One-week $100 \mathrm{mg}$ doxycycline twice daily was subsequently recommended as the local first-line treatment for rectal CT.

Aim(s)/objectives To re-evaluate the efficacy of doxycycline therapy in the treatment of rectal CT.

Methods Data was retrospectively collected on all patients diagnosed with rectal CT from $1^{\text {st }}$ October 2010 to $1^{\text {st }}$ October 2013 at a large, inner city sexual health clinic. Information was collected on gender, concurrent sexually transmitted infection (STI), treatment received, adherence to antibiotic, risk of reinfection and 4-week test of cure (TOC). Assessment of risk of re-infection included completion of telephone follow-up, verification of contact tracing of regular partners and absence of unprotected sexual intercourse.

Results 959 patients were diagnosed with rectal CT during the study period. $660(68.8 \%)$ patients received doxycycline therapy in line with local treatment protocol. TOC was performed in $473(71.7 \%)$ patients, of which $22(4.7 \%)$ were positive. Risk of re-infection was excluded in 5 cases $(22.7 \%)$ and considered possible treatment failures.

Discussion/conclusion The treatment failure rate of doxycycline for rectal CT identified in this study is similar to that reported with azithromycin and is contradictory to our previous findings. The longer study period with larger study population may explain this result. These findings suggest that TOC following treatment of rectal CT is necessary and would not support preferential use of doxycycline over azithromycin.

\section{Category: Clinical case reports}

\section{P34 TWO CASES OF ACUTE HEPATITIS E CAUSING A TRANSIENT TRANSAMINITIS IN HIV INFECTED MSM}

David Lawrence*, Yvonne Gilleece, Amanda Clarke, Martin Fisher, Daniel Richardson. Lawson Unit, Brighton and Sussex University Hospitals NHS Trust, Brighton, UK

\subsection{6/sextrans-2015-052126.78}

Background/introduction Hepatitis E Virus (HEV) is increasing in incidence. Transmission routes include faecal-oral, blood and zoonotically. Patients present with no symptoms; elevated liver enzymes; acute/chronic hepatitis and/or neuropathy. Evidence suggests poorer outcomes among HIV+ patients.

Aim(s)/objectives To describe known cases of HEV/HIV co-infection within a cohort of $2200 \mathrm{HIV}+$ patients.

Methods We present two cases.

Results Patient-1, a 63-year-old asymptomatic MSM with a 22year history of HIV, recently re-started Truvada/darunavir/ritonavir: CD4 393(17\%) cells/mm $3 \mathrm{~m}^{3}$ and HIV VL 327,824 copies/ml. Routine bloods identified newly elevated ALT 477 IU/L: other liver function, clotting and liver ultrasound were normal. He had no STIs diagnosed in the preceding year nor risk factors for HEV. A hepatitis screen was performed. HEV $\operatorname{IgG}, \operatorname{IgM}$ and PCR were positive. Treatment was supportive, with normalisation of ALT and negative HEV-PCR after eight weeks. Patient-2, a 41-year-old asymptomatic MSM with an 11-year history of HIV was ART naive: CD4 682(25\%) cells $/ \mathrm{mm}^{3}$ and HIV VL 13,109 copies/ml. Routine bloods identified newly elevated ALT $459 \mathrm{IU} / \mathrm{L}$ : other liver function, clotting and liver ultrasound were normal. He had no STIs diagnosed in the preceding year 